Bruno Schaub ${ }^{\mathrm{a}, \mathrm{b}, *}$

${ }^{a}$ Centre Pluridisciplinaire de Diagnostic Prénatal de Martinique, Centre Hospitalier et Universitaire de Martinique, Fort de France, France

'Service de Gynécologie Obstétrique, Maison de la Femme de la Mère et de l'Enfant, Centre Hospitalier Universitaire de Martinique,

Fort de France, France

Alice Monthieux

Service de Gynécologie Obstétrique, Maison de la Femme de la Mère et de l'Enfant, Centre Hospitalier Universitaire de Martinique, Fort de France, France

Fatiha Najihoullah Service de Virologie, Centre Hospitalier Universitaire de Martinique, Fort de France, France

Caroline Harte

Service de Gynécologie Obstétrique, Maison de la Femme de la Mère et de l'Enfant, Centre Hospitalier Universitaire de Martinique, Fort de France, France

Raymond Césaire Service de Virologie, Centre Hospitalier Universitaire de Martinique, Fort de France, France

Eugénie Jolivet ${ }^{\mathrm{a}, \mathrm{b}}$ Jean-Luc Voluménie ${ }^{\mathrm{a}, \mathrm{b}}$

${ }^{a}$ Centre Pluridisciplinaire de Diagnostic Prénatal de Martinique, Centre Hospitalier et Universitaire de Martinique, Fort de France, France

${ }^{\mathrm{b}}$ Service de Gynécologie Obstétrique, Maison de la Femme de la Mère et de l'Enfant, Centre Hospitalier Universitaire de Martinique, Fort de France, France

*Corresponding author at: Centre Pluridisciplinaire de Diagnostic Prénatal de Martinique, Maison de la Femme de la Mère et de l'Enfant, Centre Hospitalier et Universitaire de Martinique, BP 632, 97261 Fort-de-France, Martinique, France. Fax: +596 596750530 E-mail address: bruno.schaub@chu-fortdefrance.fr (B. Schaub).

Received 14 October 2016

http://dx.doi.org/10.1016/j.ejogrb.2016.10.041

\section{Outcomes of vaginal birth after caesarean: experience of a Portuguese hospital}

\section{Dear Editors,}

In 2009, Portugal had a peak increase on caesarean rate to $32.9 \%$ [1], with no positive impact on maternal or neonatal mortality, being far from the World Health Organization recommendation of 15\%. A trial of labor after caesarean (TOLAC) provides an option to reduce morbidity in women who seek larger families and, when successfully achieved, vaginal birth after caesarean (VBAC) yields less morbidity than a programmed repeated caesarean. In order to offer proper counsel to women in the pre-natal period we reviewed all VBAC in our Institution from January 2014 until December 2015 (Table 1).

Overall, VBAC represented $4.1 \%(n=114$; Table 1$)$ of all vaginal deliveries and although the reported induction rate was $22.8 \%$ it did not add any major maternal or neonatal morbid-mortality which could be related to the fact that the sample is not large enough to discern a difference in rare events such as uterine rupture or neonatal asphyxia. In our review the labor induction group had a higher percentage, although not statistically significant $(p=0.140)$, of women with previous vaginal delivery $(21.7 \%$ versus $10.2 \%$ ) which we hypothesize to have had a positive impact on obstetrical outcomes. Besides Bishop Score and a past of previous vaginal delivery the risk of uterine rupture in labor induction seems to be also dependent on the induction method of choice. In our population prostaglandin $\mathrm{E}_{2}\left(\mathrm{PE}_{2}\right)$ was the most commonly used (20.2\%). Between induction methods, oxytocin adds the lowest risk of uterine rupture (1.1\%) [2], nevertheless and according to Cahill et al. (2008) [3] multicentre, retrospective cohort study of 25000 women, the risk seems to be dose

Table 1

Global evaluation of VBAC $(n=114)$

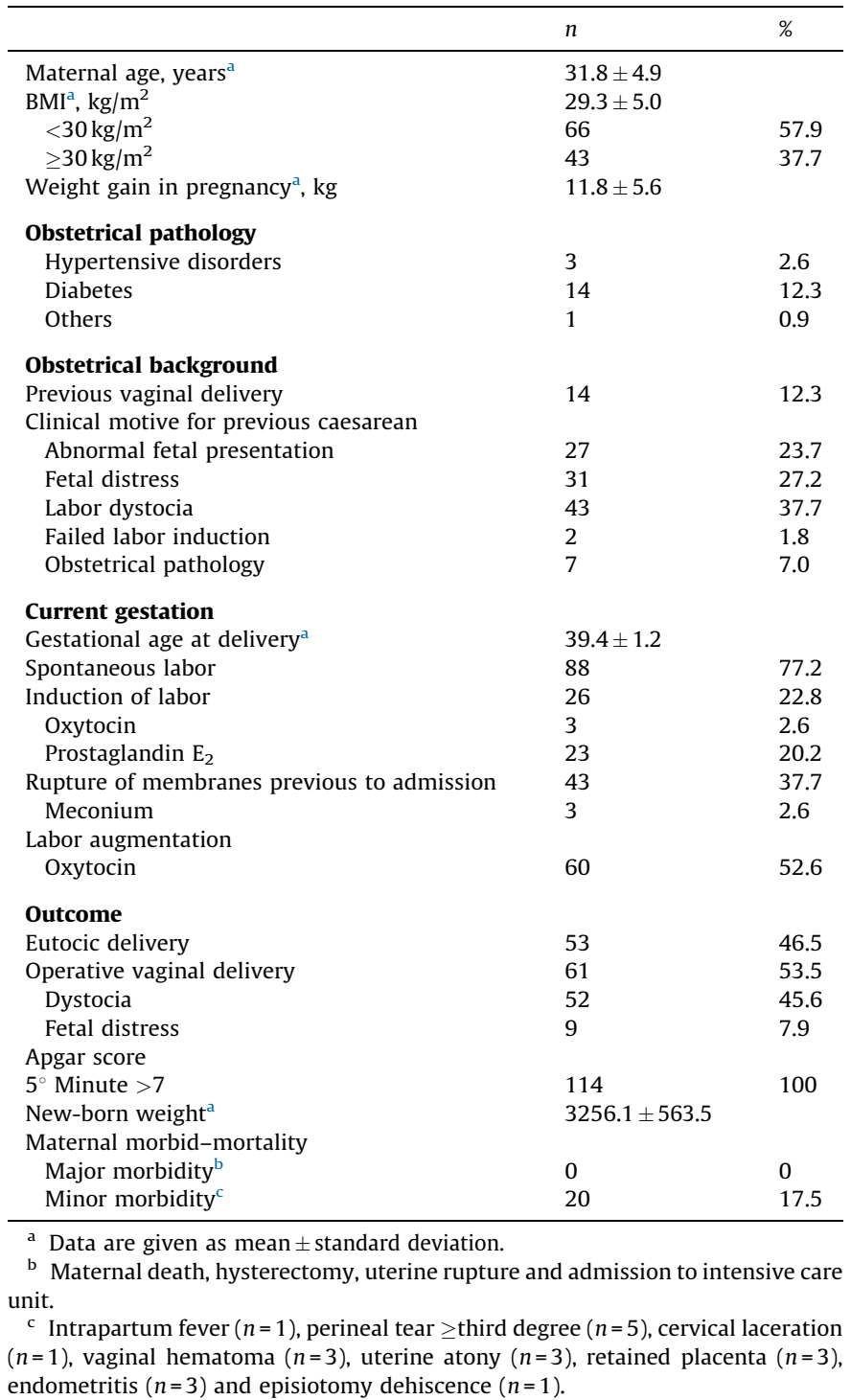


dependent with an estimated incidence of $2.9 \%$ and $3.6 \%$ for doses above 20 and $30 \mathrm{mU} / \mathrm{min}$, respectively. Labor induction with prostaglandins is associated with $1.4 \%$ [2] risk of rupture. Prostaglandin $\mathrm{E}_{1}$ is not currently recommended since Wing et al. (1998) trial of 17 patients showed a rate of uterine longitudinal rupture of $11.8 \%$ [4]. Although the clinical use of $\mathrm{PE}_{2}$ remains controversial in our review it was not associated to uterine rupture however besides the small sample size another weakness is the lack of Bishop Score since among studies it remains unclear whether the increased risk of rupture is due to an unfavourable bishop or the method of induction itself.

In a secondary analyses obese women had no increased rates of operative vaginal delivery or associated morbid-mortality nevertheless this should be interpreted with caution since obesity group had a higher proportion of women with a prior vaginal delivery ( $18.6 \%$ versus $6.1 \% ; p=0.042$ ). Interestingly obese women had a smaller proportion of women with spontaneous labor $(65.1 \%$ versus $84.8 \% ; p=0.017$ ) and delivered in a later gestational age (39.8 \pm 1.0 versus $39.2 \pm 1.2 ; p=0.011$ ) which might be related to poorer uterine contractility. According to published data, obesity is associated with high cholesterol levels which modifies the viscosity and membrane fluidity with a consequent disturbance in calcium intracellular transport leading to diminished uterine contractility seen is obese [5].

Throughout our study we found no predictive factors of operative VBAC including maternal and gestational age, induction of labor or clinical indication of previous caesarean.

When attempting VBAC, obstetrical characteristics should be taken into account for patient counseling regarding the risks and benefits of TOLAC. Although the use of $\mathrm{PE}_{2}$ showed to be safe we were not able to correlate it with an initial cervical examination. Nevertheless, when facing a favorable Bishop Score, induction of labor should be seriously considered. To the best of our knowledge, an unfavourable cervical examination enhances the risk of uterine rupture prompting the consideration for the need of mechanical cervical methods that ripen the cervix with no immediate uterine contractility associated.

\section{References}

[1] Direção Geral da Saúde. Indicadores e metas do PNS. http://impns.dgs.pt/ nascer-com-saude/ [accessed 08.06.16].

[2] Landon MB, Hauth JC, Leveno KJ, et al. Maternal and perinatal outcomes associated with a trial of labor after prior caesarean delivery. National Institute of Child Health and Human Development Maternal-Fetal Medicine Units Network. N Engl J Med 2004;351:2581-9.

[3] Cahill AG, Waterman BM, Stamilio DM, et al. Higher maximum doses of oxytocin are associated with an unacceptably high risk for uterine rupture in patients attempting vaginal birth after cesarean delivery. Am J Obstet Gynecol 2008;199. 32.e1.

[4] Wing DA, Lovett K, Paul RH. Disruption of prior uterine incision following misoprostol for labor induction in women with previous caesarean delivery. Obstet Gynecol 1998;91:828.

[5] Wray S, Jones K, Kupittayanant S, et al. Calcium signalling and uterine contractility. J Soc Gynecol Investig 2003;10:252-64.

Vania Costa Ribeiro ${ }^{\mathrm{a}, \mathrm{b}, *}$ ${ }^{a}$ Centro Hospitalar Tondela-Viseu, Hospital São Teotónio, Departamento de Ginecologia-Obstetrícia, Viseu, Portugal ${ }^{\mathrm{b}}$ Centro Hospitalar Lisboa Central, Maternidade Dr. Alfredo da Costa, Lisbon, Portugal

Nuno Nogueira Martins Francisco Nogueira Martins Centro Hospitalar Tondela-Viseu, Hospital São Teotónio, Departamento de Ginecologia-Obstetrícia, Viseu, Portugal
${ }^{*}$ Corresponding author at: Centro Hospitalar Lisboa Central, Maternidade Dr. Alfredo da Costa, Lisbon, Portugal E-mail address: dokvcr@gmail.com (V. Costa Ribeiro).

Received 3 October 2016

http://dx.doi.org/10.1016/j.ejogrb.2016.10.040

\section{Use of a laparoscopic specimen retrieval bag for delivery of an entrapped head in a breech fetus at 21 weeks gestation}

\section{Dear Editors,}

A 29 years old nulliparous patient presented to delivery suite at 21 weeks gestation complaining of vaginal bleeding. She was low risk at booking except for a BMI of 31, and had routine antenatal care. A speculum examination was performed which showed blood stained liquor. The patient was admitted and an ultrasound scan revealed a live fetus with oligohydramnios and absent end diastolic flow on Doppler. The patient and her husband were fully counselled by a consultant about the risks of continuing the pregnancy versus elective termination. The couple suffered from infertility, and had been trying for 9 years. There were no signs of labour. They therefore requested $24 \mathrm{~h}$ to consider their options, during which they remained on the antenatal ward.

Eight hours later the patient's husband requested assistance from the midwife caring for her. On arrival in the room the midwife noted feet protruding from the vagina. The oncall registrar attended and found the fetus delivering as a breech, with the umbilicus at the level of the introitus. The patient was encouraged to push, but no further descent of the body occurred. Vaginal examination revealed the cervix was only partially dilated with both arms stuck in the cervix behind the fetal head. The arms were gently delivered and the patient was again encouraged to push. The fetus remained undelivered, and further examination confirmed head entrapment. The cervix could not be encouraged to stretch either with pushing or gentle manipulation with fingers. Gentle traction on the fetus caused tearing of the skin at the junction of the neck and shoulders.

The patient was transferred to theatre and a general anaesthetic was administered. As the fetus was non-viable and the couple had long standing fertility issues it was important to deliver an intact fetus as the patient wished to view it, yet we did not want to cause any potential long term complications to the mother. It was therefore decided we should avoid Dührson's Incisions and caesarean section/hysterotomy if at all possible.

We therefore passed a specimen retrieval bag, usually used during laparoscopic surgery, between the fetal head and the operator's index finger. The retrieval bag was rotated within the uterus so that it opened next to the fetal head. The aperture was moved so that it was enclosing the fetal head and the bag drawn closed in the usual way. Gentle traction to the bag effected pressure to the top of the head rather than the shoulders allowing the head to gently dilate the cervix and the fetus to be delivered successfully with no cervical trauma.

The fetus was delivered intact with no obvious injuries from the specimen retrieval bag. Careful examination of the mother's cervix and vagina also revealed no evidence of trauma from the technique.

The family were given the opportunity to view and grieve for an intact fetus, which is important as an opportunity to bond is now routine practice in the management of late miscarriages and still births. 